\title{
IN MEMORIAM: JOHN H. CONWAY
}

\author{
MICHEL BROUÉ
}

"Conway was arguably an extreme point in the convex hull of all mathematicians" wrote Terry Tao after John H. Conway died from COVID-19 on April 11, 2020. Emeritus professor at Princeton University, which he joined in 1986 after spending almost 20 years at Cambridge University (U.K.), Conway was 82 years old.

"An extreme point...". Indeed, Conway has contributed to so many aspects of mathematics, including finite group theory, number theory, game theory, tiling, logic, knot theory, combinatorics, algorithmics. He also invented a profusion of games, such as "Phutball". He said he was proud of "Surreal Numbers" (an enormous new world of numbers) and "Free Will Theorem" (a sort of mathematical formulation of the philosophical notion of free will, inspired by quantum mechanics). It seems he is most widely known (which he sometimes regretted) as the inventor of the "Game of Life", the theory of cellular automata-indeed of deterministic behavior, quite unlike "Free Will Theorem": "I have not become a world expert on one topic, I don't do that. I am just interested in a lot of things," said he.

In 1970, I started my PhD under Claude Chevalley, who suggested that I should consult Jean-Pierre Serre for a good problem. Serre told me that a good start might consist in reading an interesting new paper and trying to understand it with my own ways; he said, "You might read the paper of Conway about the automorphisms group of the Leech lattice". Which I did, since Chevalley confirmed, "Serre is always right". I found that paper-both the object and its symmetries studied there - just fantastic, and not that difficult to understand. Harder to understand was the tour de force Conway produced to arrange what appeared to me at first as a complicated combinatorial mess into the magic organisation of his famous Conway group. As expected by Serre, this mathematical jewel launched my work. In 1972, I visited John Thompson in Cambridge, and I met John Conway for the first time. He frightened me. Too tricky, too quick, too smart for me, playing all the time, surrounded by a troop of young mathematicians who seemed to understand so much better than me; he was a brilliant prestidigitator, a math juggler.... I dared not really interact with him, I needed stable ground, and he was the "Peter Pan of Maths". Fortunately, I was less shy when I met him later in Luminy and in 2015 in Princeton for "celebrating thirty years of the Atlas of Finite Groups and honoring John Conway". The Atlas of Finite Groups (1985) was indeed one of the big achievements of this extraordinary Cambridge group of mathematicians around Conway; in particular, it contains the character tables of all sporadic finite simple groups.

The construction of the Conway group (actually providing three new nonabelian finite simple groups) played a central role in the classification of finite simple groups. The Conway group was at the heart of the subsequent discovery and construction 
of the largest of the sporadic simple groups, the "Monster", for which the Conway group looks like a sort of Weyl group. The smallest dimension to faithfully represent that Monster is 196883 . It happens that $196883+1=196884$, a fact noticed by John McKay, who knew the Fourier development of the $j$-invariant,

$$
j=\frac{1}{q}+196884 q+21493760 q^{2}+\cdots .
$$

After computing other coincidences between that Fourier expansion and degrees of representations of the Monster, McKay drew the attention of John Thompson. Thompson, imagining the existence of a graded module endowed with an action of the Monster $M$, suggested computing the functions $j_{g}$ defined by the graded traces of some $g \in M$. In a famous and intriguing paper entitled Monstrous Moonshine, Conway and Norton published computations of low-order terms of such graded traces, and they conjectured the existence of a graded $\mathbb{C} M$-module such that the graded traces of $g$ define functions $j_{g}$ that are Hauptmoduln for appropriate subgroups $\Gamma(g)$ of $\mathrm{SL}_{2}(\mathbb{R})$ : they generate the field of meromorphic functions on the quotient of the upper complex half-plane by $\Gamma(g)$. That conjecture was proved in 1992 by Richard Borcherds, one of Conway's PhD students.

Conway is also widely known as a co-author of the impressive book, Winning Ways for Your Mathematics Plays, as well as for writing the classic, Sphere Packings, Lattices and Groups, with Neil Sloane. He increased the average of the Erdös number, having had more than 70 coauthors. He was known for his eccentricities, wearing sport short pants at the ceremony of the Academy for Arts and Sciences, or ending his address to the International Congress of Mathematicians in 1994 by laying on the floor and waggling his arms and legs. He was also the main topic of a nice biography written while he was alive, Genius at Play, The Curious Mind of John Horton Conway, by Siobhan Roberts.

Université de Paris, Paris, France 